\title{
Sistema de suporte à decisão para recomendação de uso e manejo da terra
}

\author{
Monica L. Giboshi', Luiz H. A. Rodrigues ${ }^{2} \&$ Francisco Lombardi Neto ${ }^{3}$
}

\begin{abstract}
RESUMO
Objetivou-se, com este trabalho, o desenvolvimento de um sistema de apoio à decisão para a recomendação de uso e manejo da terra, integrando Sistema Especialista, Sistema de Informações Geográficas, uma base de dados e uma interface para interpretar a entrada de dados do usuário e as mensagens passadas entre os subsistemas. O sistema desenvolvido determina a capacidade de uso da terra; recomenda usos adequados para cada classe de capacidade de uso como também práticas de conservação e manejo do solo e identifica áreas de conflito comparando os mapas de uso da terra com o mapa de capacidade de uso. Todos os resultados podem ser visualizados em janelas do programa, gravados ou impressos em forma de relatório. A área utilizada para testar o sistema é o Município de Santo Antônio do Jardim, SP. O sistema mostrou ser uma ferramenta poderosa e eficaz, permitindo avaliar-se uma região, oferecendo suporte para uma tomada de decisão mais fundamentada.
\end{abstract}

Palavras-chave: sistemas especialistas, sistema de informações geográficas, capacidade de uso da terra, conservação do solo

\section{A decision support system for recommending land use and soil management}

\begin{abstract}
The objective of this work was to develop a decision support system to recommend land use and practices for soil conservation and management, which integrates an Expert System, Geographic Information System (GIS), a database and an interface to monitor input and output data and the messages passed between the subsystems. Using soil and slope maps, the developed system determines land capability from information stored in the database and also supplied by SIG; it recommends adequate uses for land capability class as well as practices for soil con

servation and management and identifies conflict areas comparing the maps of land use with the land capability. All results can be visualized by the user through windows of the program, recorded or printed in form of report. In order to test the system, the municipality of Santo Antônio do Jardim, in the State of São Paulo, Brazil, was selected. The system is a powerful and efficient tool, permitting the evaluation of a region and thereby offering support for adequate decision making.
\end{abstract}

Key words: expert system, geographic information system, land capability, soil conservation.

1UNIPINHAL/Engenharia Ambiental, Av. Hélio Vergueira Leite, s/n - CEP 13990-000, Espírito Santo do Pinhal, SP. E-mail: mlgiboshi@hotmail.com ${ }^{2}$ FEAGRI/UNICAMP, CP 6011, CEP 13083-875, Campinas, SP. Fone: (19)3788-1062, E-mail: lique@agr.unicamp.br

3IAC, Rua Regente Feijó 744. CEP 13400-100, Piracicaba, SP. E-mail: flombardi43@yahoo.com.br 


\section{INTRODUÇÃO}

O solo é um recurso natural que demora milhares de anos para se formar e que se pode degradar, muitas vezes até de forma irreversível, em algumas poucas décadas (ou mesmo em alguns anos) por sua má utilização pelo homem. Da mesma forma, os processos de degradação do solo também podem provocar a poluição dos recursos hídricos, quer seja pela quantidade de sedimentos produzidos por meio de processos erosivos, quer pela movimentação de produtos químicos. É necessário, portanto, evitar que esses processos de degradação ambiental ocorram e, se eles já estiverem instalados, deverão ser interrompidos ou controlados.

Um elemento chave nos programas de conservação do solo e da água é a seleção de medidas que reduzam a erosão, principalmente em áreas com agricultura intensiva. Um programa de conservação do solo consiste na utilização da terra, de acordo com a sua capacidade e na aplicação correta de práticas de conservação para garantir seu uso intensivo e permanente sem provocar sua degradação.

Segundo Bertolini \& Lombardi Neto (1994), o desgaste e o empobrecimento do solo nas suas diversas fases e formas, podem ser evitados com a utilização de práticas que aumentam a cobertura vegetal e a infiltração da água no perfil do solo e reduzem o escoamento superficial. Desta forma, obtémse, como reflexo, uma melhoria da quantidade e qualidade das águas, além da preservação da vida silvestre e melhoria do ambiente.

Portanto, antes de se adotar um programa de conservação do solo é necessário determinar a capacidade de uso da terra, que permite estabelecer bases para o seu melhor aproveitamento sem sofrer depauperamento pelos fatores de degradação.

A capacidade de uso da terra pode ser conceituada como a adaptabilidade da terra às diversas formas de utilização agrícola, sem que ocorra o depauperamento do solo pelos fatores de desgaste e empobrecimento, através do seu uso (Lepsch et al., 1991). As classes de capacidade de uso representam um grupamento de terras com o mesmo grau de limitação, definindo condições de aproveitamento e riscos de degradação semelhantes. São designadas por algarismos romanos de I a VIII, e quanto maior o seu valor maior é a restrição ao uso.

A determinação da capacidade de uso da terra envolve a interpretação dos fatores que têm maior influência sobre o uso da terra, como a natureza do solo, a declividade e a erosão, entre outros; é uma tarefa complexa que envolve conhecimentos diversos e interdisciplinares, além de uma vasta quantidade de dados, principalmente quando se trata de uma área grande, tornando este trabalho mais difícil e demorado.

Um Sistema de Suporte à Decisão (SSD) é um sistema baseado em computador, projetado para aumentar a efetividade dos tomadores de decisões; possui funções específicas que permitem buscar informações e fornecer subsídios para o processo de tomada de decisão, que se desenvolve através da interação constante do usuário com um ambiente especialmente criado para dar subsídio às decisões a serem tomadas (Sprague \& Watson, 1989).
Sistemas especialistas e sistemas de informações geográficas são ferramentas que podem ser integradas em um SSD, uma vez que têm a capacidade de armazenar e processar uma vasta quantidade de informações.

Sistemas especialistas são programas computacionais que permitem a sistematização da lógica utilizada por especialistas, em suas áreas de domínio, ou seja, “imitam” o raciocínio utilizado por especialistas ao resolverem problemas (Plant \& Stone, 1991). Têm a capacidade de armazenar conhecimentos necessários para avaliar e resolver problemas e, também, fazer recomendações.

Todo sistema especialista é formado por três componentes básicos: uma base de conhecimento do domínio, um sistema ou mecanismo de inferência e uma interface com o usuário. A base de conhecimento do sistema especialista contém a representação do conhecimento do domínio do problema. O mecanismo ou sistema de inferência aplica as regras de lógica racional para pesquisar a base de conhecimento em busca de soluções. A interface com o usuário é conhecida como o sistema de entrada e saída que permite a comunicação bidirecional, isto é, a troca de informações entre o usuário e o mecanismo de inferência (Sawyer \& Foster, 1986).

Sistemas de informações geográficas podem armazenar, manipular, transformar, analisar e exibir informações georreferenciadas, contidas em mapas e/ou bancos de dados, gerando novas informações (Burrough, 1986); podem ser utilizados em estudos de mudança de uso da terra, para a avaliação de terras e em estudos de degradação do solo, entre outros.

Com este trabalho, tem-se como objetivo descrever e avaliar um sistema de suporte à decisão para recomendação de uso e manejo da terra, utilizando-se as técnicas citadas.

\section{MATERIAL E MÉTODOS}

O sistema é composto por um conjunto de subsistemas: dois sistemas especialistas (SE), um para determinar a capacidade de uso da terra e outro para fazer as recomendações das práticas de conservação e manejo do solo; um Sistema de Informações Geográficas (SIG); uma Base de Dados e uma Interface. Cada um desses subsistemas é um programa individual que se comunica com os outros através da interface.

Os SEs foram desenvolvidos com o uso de uma linguagem de alto nível, conhecida como CLIPS (C Language Integrated Production System), desenvolvida por Software Technology Branch, NASA/Lyndon B. Johnson Space Center (Giarratano \& Riley, 1993).

Para determinar a capacidade de uso da terra além da declividade (Tabela 1), consideram-se os seguintes fatores limitantes ao uso da terra: pedregosidade (pd), rochosidade (r), risco de geada (g), risco de inundação (i), profundidade efetiva (p), drenagem interna (d), disponibilidade de água (w), risco de erosão (re), restrição à mecanização $(\mathrm{m})$, disponibilidade de nutrientes (v), toxicidade por alumínio (a) e fixação de fósforo (f). Para cada fator é determinado um grau de limitação ao uso (0: nulo; 1: ligeiro; 2 : moderado; 3 ; forte e 4 : muito forte); sua combinação em conjunto com a declividade fornece a classe de capacidade de uso da terra (Giboshi, 1999). 
Tabela 1. Classes de declividade

\begin{tabular}{cc}
\hline Classe de declividade & Declividade (\%) \\
A & $0-3$ \\
B & $3-6$ \\
C & $6-9$ \\
D & $9-12$ \\
E & $12-18$ \\
F & $18-25$ \\
G & Maior que 25 \\
\hline
\end{tabular}

São oito classes de capacidade de uso, numeradas com algarismos romanos de I a VIII, segundo Lepsch et al. (1991).

As classes de capacidade de uso são apresentadas de forma diferente do "Manual para Levantamento Utilitário do Meio Físico e Classificação de Terras no Sistema de Capacidade de Uso", de Lepsch et al. (1991); são representadas por algarismos romanos, seguidas dos símbolos dos fatores limitantes acompanhados dos seus respectivos graus de limitação ao uso da terra e em ordem decrescente; por exemplo, para o Latossolo Vermelho (LEd3/so), unidade Limeira, na declividade entre 3 e $6 \%$, com classe de capacidade de uso da terra III v3 a3 f2 re1 m1, tem-se: Classe III de capacidade de uso da terra, apresentando disponibilidade de nutrientes (v) com limitação forte (3), toxicidade por alumínio (a) com limitação forte (3), fixação de fósforo com grau moderado (2), risco de erosão (re) ligeiro (1) e com ligeira restrição (1) à mecanização (m). Quando o fator não aparece, significa que seu grau de limitação é nulo.

Outra modificação introduzida foi a simbologia "VIIIff" para áreas que devem ser preservadas por fragilidade ambiental, decorrente de condições especiais de solo e/ou relevo e/ou clima, e "VIIIFF" para áreas de preservação ambiental protegidas por lei, de forma semelhante a Pereira (2002).

As recomendações de uso da terra e manejo são feitas por um sistema especialista que utiliza as regras que, para isto, compõem sua base de conhecimento. Essas regras foram elaboradas com base em literatura existente sobre o assunto (Bertoni \& Lombardi Neto, 1990; Lombardi Neto \& Drugowich, 1994; Lepsch et al., 1991; Lombardi Neto \& Bellinazzi Júnior, 1989; Sartori, 2004) e entrevistas realizadas com especialistas da área.

Os usos recomendados pelo sistema, são: a) cultura anual; b) cultura perene; c) pastagem; d) reflorestamento; e) área de preservação ambiental; f) área de uso turístico e g) área de conservação hídrica.

Para as classes de capacidade de uso de I a IV, o sistema recomenda o uso de cultura anual e/ou perene e, também, define se o uso é adequado, moderado ou restrito, pois quanto maior for a classe mais complexos serão os problemas de conservação. O uso com pastagem é recomendado para as classes V, VI e VII; reflorestamento, para as classes VI e VII; e a classe VIII deve ser utilizada como área de preservação e/ou uso turístico e/ou, ainda, área de conservação hídrica.

O SIG utilizado neste trabalho foi o Idrisi, versão 32, que foi integrado ao sistema de suporte à decisão para gerar o mapa com a classificação com a capacidade de uso da terra, e outro mostrando a adequação de uso da terra. O SIG também foi utilizado para calcular a área correspondente a cada classe de capacidade de uso, assim como a área de adequabilidade de uso.

A interface foi desenvolvida em Visual Basic, versão 5.0, e é responsável pelo gerenciamento de todo o sistema como, por exemplo, a apresentação de janelas, menus, caixas de diálogo, mapas e tabelas na tela do monitor, além da impressão e/ou gravação dos resultados obtidos. Todos os diálogos entre o usuário e o SSD são feitos através da interface, assim como a comunicação entre os componentes do sistema desenvolvido.

A base de dados do sistema contém informações dos solos, como: textura; teores médios de argila, silte, areia fina e areia grossa, CTC, saturação por bases, saturação por alumínio, erodibilidade, drenagem, presença de pedregosidade e/ ou rochosidade, profundidade efetiva etc.

A área utilizada para testar o sistema é o Município de Santo Antônio Jardim, SP, com uma área de 10.875 ha. Os solos que ocorrem no município são Latossolos, Argissolos e Cambissolo, representados por seis unidades simples de mapeamento e duas associações de classe de solos (Tabela 2), segundo levantamento pedológico semidetalhado de Oliveira (1992).

Solicitou-se a quatro especialistas que fizessem a recomendação de uso e das práticas de conservação e manejo do solo, em função da capacidade de uso da terra.

Os especialistas que participaram da validação: Dra. Isabella Clérici De Maria (Centro de Solos e Recursos Ambientais, Instituto Agronômico de Campinas); Dr. Lauro Charlet Pereira (Embrapa Meio Ambiente); Dra. Mara Marinho Weill (Faculdade de Engenharia Agrícola da UNICAMP) e Dr. Pedro Luiz Donzelli (do Centro de Solos e Recursos Ambientais, Instituto Agronômico de Campinas).

\section{RESULTADOS E DISCUSSÃO}

O primeiro resultado apresentado pelo sistema é o relatório com a capacidade de uso da terra, o qual pode ser apenas visualizado e/ou gravado como um arquivo no editor de textos (Figura 1). O sistema também apresenta um mapa (Figura 2) com as classes de capacidade de uso da terra, juntamente com uma tabela que mostra a área em hectares para cada classe.

As terras passíveis de utilização com cultivo (anual e/ou perene), pastagem e reflorestamento, representam $37 \%$ (4024,44 ha) do município e correspondem às classes II, III e IV de capacidade de uso. O município possui 42,7\% (4648,41 ha) de terras impróprias para cultivo porém adaptadas para pastagem ou reflorestamento, que correspondem às classes V, VI e VII de capacidade de uso. Também há uma área correspondente às classes VIIIff e VIIIFF, representando 19,5\% (21,24 ha) do município, com terras impróprias para qualquer tipo de cultivo, pastagem ou reflorestamento e que devem ser utilizadas como áreas de preservação ambiental ou de conservação hídrica ou, ainda, para fins turístico. 
Tabela 2. Solos do município de Santo Antônio do Jardim

\begin{tabular}{|c|c|c|c|c|}
\hline \multicolumn{3}{|c|}{ Semidetalhado (Oliveira, 1992) } & \multicolumn{2}{|c|}{ SBCS $^{1}$ (EMBRAPA, 1999) } \\
\hline Classificação & Símbolo & Unidade & Classe & Símbolo \\
\hline Latossolo Vermelho Escuro & LEd3 & Limeira & Latossolo Vermelho & LV \\
\hline Latossolo Vermelho-Amarelo & LVd3 & Mato Dentro & Latossolo Vermelho-Amarelo & LVA \\
\hline Latossolo Vermelho-Amarelo & LVd8 & Camarguinho & Latossolo Vermelho-Amarelo & LVA \\
\hline Podzólico Vermelho-Amarelo & PV5 & Cristalino & Argissolo Vermelho-Amarelo & PVA \\
\hline $\begin{array}{l}\text { Podzólico Vermelho-Amarelo } \\
\text { com cascalhos }\end{array}$ & PV5c & $\begin{array}{l}\text { Cristalino com } \\
\text { cascalho }\end{array}$ & Argissolo Vermelho-Amarelo & PVA \\
\hline Cambissolo & $\mathrm{C} 1 / \mathrm{p}$ & Sete Lagoas & Cambissolo & C \\
\hline $\begin{array}{c}\text { Associação de Latossolo Vermelho-Amarelo } \\
+ \\
\text { Podzólico Vermelho-Amarelo Latossólico }\end{array}$ & $\begin{array}{l}\mathrm{LVd} 4 \\
+ \\
\mathrm{PVL}\end{array}$ & $\begin{array}{c}\text { Ponte Funda } \\
+ \\
\text { Cristalino }\end{array}$ & $\begin{array}{c}\text { Latossolo Vermelho-Amarelo } \\
+ \\
\text { Argissolo Vermelho-Amarelo }\end{array}$ & $\begin{array}{l}\text { LVA } \\
+ \\
\text { PVA }\end{array}$ \\
\hline $\begin{array}{c}\text { Associação de Podzólico Vermelho-Amarelo Latossólico } \\
++ \\
\text { Podzólico Vermelho-Amarelo }\end{array}$ & $\begin{array}{l}\text { PVL } \\
+ \\
\text { PV5 }\end{array}$ & $\begin{array}{l}\text { Latossólico } \\
+ \\
\text { Cristalino }\end{array}$ & Argissolo Vermelho-Amarelo & PVA \\
\hline
\end{tabular}

1 - Sistema Brasileiro de Classificação de Solos (EMBRAPA, 1999)

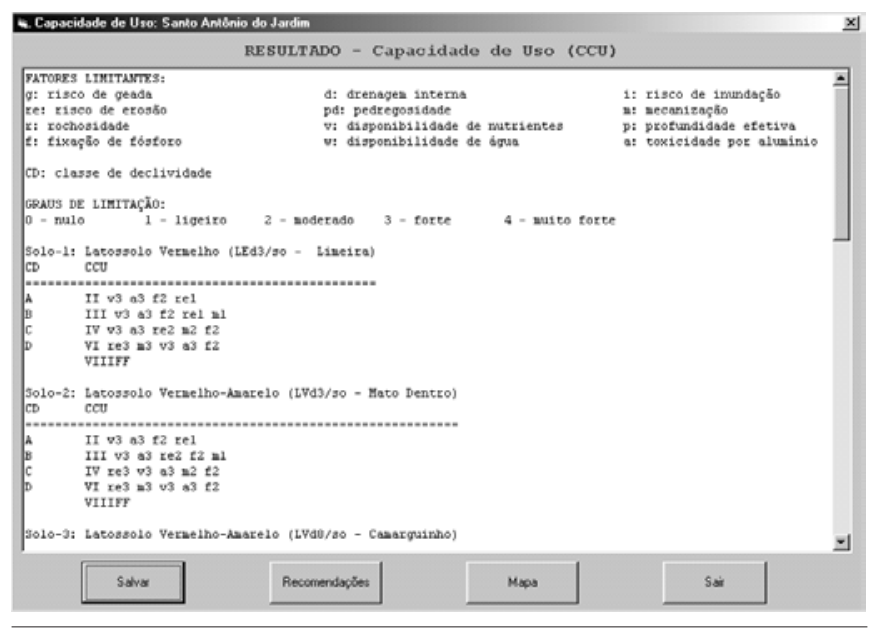

Figura 1. Janela do programa com a capacidade de uso da terra

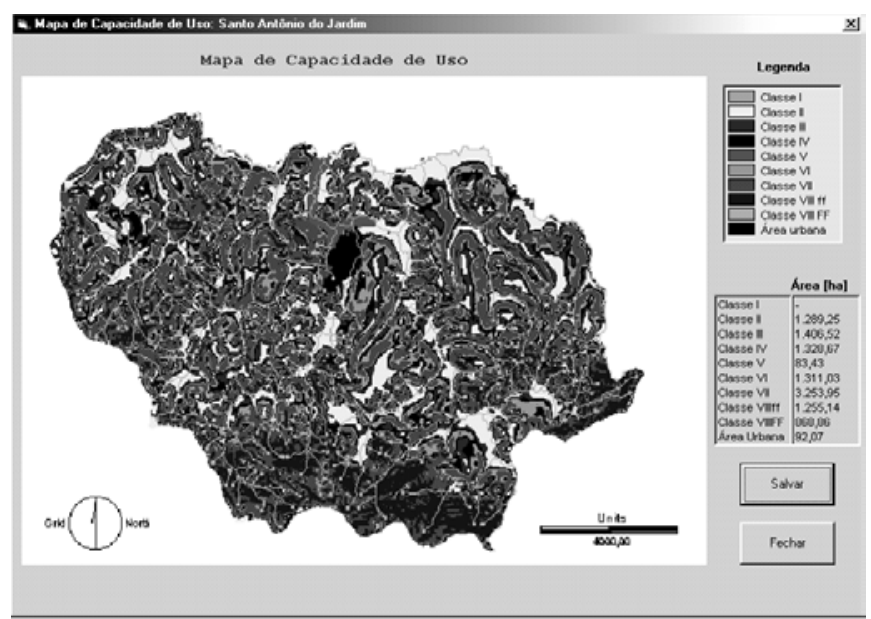

Figura 2. Mapa com as classes de capacidade de uso da terra, apresentado pelo sistema

Os resultados apresentados na Tabela 3 mostram que a principal restrição para os Latossolos está relacionada à fer- tilidade (disponibilidade de nutrientes $-\mathrm{v}$, toxicidade por alumínio - a, e fixação de fósforo - f) com grau de restrição variando entre moderado e muito forte. Outra limitação para esses solos diz respeito à erosão e restrição à mecanização, ambos com grau variando de ligeiro a forte, de acordo com a declividade.

O Argissolo Vermelho-Amarelo sem cascalho e a Associação 2 (PVL + PV5/o) têm, como principal limitação, o risco de erosão com grau de restrição entre moderado e muito forte. A restrição à mecanização varia entre ligeiro e muito forte. No que diz respeito à fertilidade, este solo apresenta restrição variando de ligeira a moderada; apresenta, também, limitação ligeira relacionada à profundidade efetiva. O Argissolo Vermelho-Amarelo com cascalho apresenta as mesmas limitações que os solos anteriores, com mais uma restrição devido à presença de cascalho, ou seja, restrição ligeira relacionada ao fator pedregosidade.

O Cambissolo apresenta limitação moderada para a drenagem interna em virtude da sua localização em planície aluvional. Em relação à fertilidade, o grau de limitação varia de ligeiro a forte. A Associação 1 (LVd4 + PVL) apresenta, como principal limitação, a fertilidade, com grau variando entre moderado e forte. A limitação à mecanização e ao risco de erosão apresenta grau variando entre ligeiro e forte.

Outro resultado apresentado pelo sistema é o mapa denominado "Adequabilidade de Uso", obtido através do cruzamento entre o mapa de capacidade de uso e o mapa com o uso atual da região. Identificaram-se quatro classes de adequação de uso: adequada, inadequada, subutilizada e não classificada, conforme os seguintes critérios:

a) Adequada se:

1) o uso atual for cultura anual ou perene e a classe de capacidade de uso entre I e IV;

2) o uso atual for pastagem e a classe de capacidade de uso igual a $\mathrm{V}$ ou $\mathrm{VI}$;

3) o uso atual for pastagem ou reflorestamento e a classe de capacidade de uso VII; 
Tabela 3. Capacidade de uso da terra para o município de Santo Antônio do Jardim

\begin{tabular}{|c|c|c|}
\hline Solo & CD & CCU \\
\hline \multirow{5}{*}{$\begin{array}{l}\text { Latossolo Vermelho } \\
\text { (LEd3/so - Limeira) }\end{array}$} & $A$ & II v3 a3 f2 re1 \\
\hline & B & III v3 a3 f2 re1 m1 \\
\hline & C & IV v3 a3 re2 m2 f2 \\
\hline & $\mathrm{D}$ & VI re3 m3 v3 a3 f2 \\
\hline & & VIIIFF \\
\hline \multirow{5}{*}{$\begin{array}{l}\text { Latossolo Vermelho-Amarelo } \\
\text { (LVd3/so - Mato Dentro) }\end{array}$} & $A$ & II v3 a3 f2 re1 \\
\hline & $\mathrm{B}$ & III v3 a3 re2 f2 m1 \\
\hline & C & IV re3 v3 a3 m2 f2 \\
\hline & $\mathrm{D}$ & VI re3 m3 v3 a3 f2 \\
\hline & & VIIIFF \\
\hline \multirow{5}{*}{$\begin{array}{l}\text { Latossolo Vermelho-Amarelo } \\
\text { (LVd8/so - Camarguinho) }\end{array}$} & $A$ & II v4 a4 re1 f1 \\
\hline & $\mathrm{B}$ & III v4 a4 re1 m1 f1 \\
\hline & C & IV v4 a4 re2 m2 f1 \\
\hline & $D$ & VI v4 a4 re3 m3 f1 \\
\hline & & VIIIFF \\
\hline \multirow{8}{*}{$\begin{array}{l}\text { Argissolo Vermelho-Amarelo } \\
\text { (PV5 - Cristalino) }\end{array}$} & $A$ & II re2 v2 p1 f1 a1 \\
\hline & $\mathrm{B}$ & III re3 v2 p1 m1 f1 a1 \\
\hline & C & IV re4 m2 v2 p1 f1 a1 \\
\hline & $\mathrm{D}$ & VI re4 m3 v2 p1 f1 a1 \\
\hline & $E$ & VII re4 m3 v2 p1 f1 \\
\hline & $\mathrm{F}$ & VII re4 m4 v2 p1 f1 \\
\hline & $G$ & VIIIff \\
\hline & & VIIFF \\
\hline
\end{tabular}

\begin{tabular}{|c|c|c|}
\hline \multirow{8}{*}{$\begin{array}{l}\text { Argissolo Vermelho-Amarelo } \\
\text { (PV5 - Cristalino com cascalho) }\end{array}$} & A & II re2 v2 pd1 p1 m1 f1 a1 \\
\hline & B & III re3 v2 pd1 p1 m1 f1 a1 \\
\hline & C & IV re4 m2 v2 pd1 p1 f1 a1 \\
\hline & $\mathrm{D}$ & VI re4 m3 v2 pd1 p1 f1 a1 \\
\hline & $\mathrm{E}$ & VII re $4 \mathrm{~m} 3 \mathrm{v} 2 \mathrm{pd} 1 \mathrm{p} 1 \mathrm{f} 1 \mathrm{a} 1$ \\
\hline & $\mathrm{F}$ & VII re4 m4 v2 pd1 p1 f1 a1 \\
\hline & G & VIIIff \\
\hline & & VIIIFF \\
\hline \multirow{3}{*}{$\begin{array}{c}\text { Cambissolo } \\
\text { (C1/p-Sete Lagoas) }\end{array}$} & A & V v3 a3 d2 re2 f1 \\
\hline & B & V re3 v3 a3 d2 m1 f1 \\
\hline & & VIIIIFF \\
\hline \multirow{5}{*}{$\begin{array}{c}\text { Associação } 1 \\
\text { (LVd4 + PVL - Ponte Funda) }\end{array}$} & A & II v3 f3 a2 re1 \\
\hline & B & III v3 f3 re2 a2 m1 \\
\hline & C & IV re3 v3 f3 m2 a2 \\
\hline & D & VI re3 m3 v3 f3 a2 \\
\hline & & VIIIFF \\
\hline \multirow{8}{*}{$\begin{array}{c}\text { Associação } 2 \\
\text { (PVL + PV5/0-Latossólico) }\end{array}$} & A & II re2 v2 p1 f1 a1 \\
\hline & B & III re3 v2 p1 m1 f1 a1 \\
\hline & C & IV re4 m2 v2 p1 f1 a1 \\
\hline & $\mathrm{D}$ & VI re4 m3 v2 p1 f1 a1 \\
\hline & $\mathrm{E}$ & VII re4 m3 v2 p1 f1 a1 \\
\hline & $\mathrm{F}$ & VII re4 m4 v2 p1 f1 a1 \\
\hline & G & VIllff \\
\hline & & VIIIFF \\
\hline
\end{tabular}

$\overline{C D}$ - Classe de declividade; CCU - Classe de capacidade de uso da terra

4) todas as áreas de uso atual com mata;

b) Inadequada se o uso atual for cultura anual ou perene e a classe de capacidade de uso entre V e VII, ou VIIIff ou VIIIFF; c) Subutilizado quando:

1) o uso atual for pastagem ou reflorestamento e a classe de capacidade de uso entre I a IV;

2) o uso for reflorestamento e a classe de capacidade de uso entre $\mathrm{V}$ e VI

d) Não classificado: área urbana e corpos d'água.

Este resultado mostrou que 48,5\% (5254,74 ha) das terras do município estão sendo utilizadas de forma adequada, são áreas ocupadas com cultura anual e/ou perene, pastagem, reflorestamento e mata nativa. Há uma parcela que representa $31,82 \%$ (3447,18 ha) da região com uso inadequado e 14,06\% (1523,07 ha) se refere à subutilização, ou seja, a terra está sendo utilizada com intensidade menor que a sua capacidade de uso. A representada por 5,62\% (608,67 ha), com a nomenclatura "Não classificada", corresponde à área urbana e corpos d'água.

As recomendações de uso e manejo da terra são feitas com base na capacidade de uso da terra e nas limitações apresentadas pelos solos. O sistema recomenda os diferentes tipos de uso, indicando o mais adequado e avisando o usuário de que, se este fizer a opção por outro tipo de uso, estará subutilizando a terra. Quando for o caso, serão feitas recomendações para o sistema de terraceamento, indicando tipos de terraços quanto à sua função e dimensão, além de recomendações para a sua manutenção, como mostra o relatório no apendice para o solo1 (LEd/So).

A validação consistiu na comparação entre as saídas dos sistemas especialistas com os resultados obtidos pelos especialistas para o cenário utilizado, que foi o município de Santo Antônio do Jardim.

Para preservar a identidade dos especialistas, estes estão citados apenas como especialistas 1, 2, 3 e 4, e essa ordem não é a mesma daquela apresentada no item Material e Métodos.

Considerando os tipos de usos recomendados pelo sistema e aqueles recomendados pelos especialistas, tem-se o seguinte resultado: uma concordância de $71,8 \%$ para o especialista $1,84,8 \%$ para os especialistas 2 e 3 e $97,8 \%$ para o especialista 4.

Quanto às recomendações, houve concordância de 94\% para o especialista $1,83,3 \%$ para o especialista 2 e $100 \%$ para o especialista 4. Como o especialista 3 sugeriu apenas que as recomendações do sistema fossem mais específicas e não discordou das recomendações feitas, optou-se por não indicar um valor, como foi feito em relação aos outros especialistas.

Uma discussão detalhada com os resultados da validação podem ser encontrados em Giboshi (2005).

Observando a capacidade de uso da terra determinada por Lagrotti (2000), ao fazer o planejamento agroambiental do município de Santo Antônio do Jardim, nota-se que as classes de capacidade de uso determinadas por este autor, assim como as restrições ao uso da terra, correspondem às obtidas com o uso do SSD apresentado. 


\section{CONCLUSÕES}

1. A integração de Sistemas Especialistas, Sistema de Informações Geográficas e Banco de Dados, mostrou tratar-se de uma técnica eficaz para o desenvolvimento de sistemas de suporte à decisão para recomendações de uso e manejo da terra.

2. O sistema desenvolvido é de fácil utilização; o usuário não precisa ser um especialista, embora deva ter algum conhecimento sobre o assunto, para levantar as informações necessárias ao funcionamento do sistema e fornecê-las corretamente em busca do bom funcionamento do mesmo.

3. O sistema permite uma visão geral da área de estudo, facilitando a identificação de áreas de conflito, através do mapa de adequabilidade de uso.

4. Verificou-se a existência de uma área razoável do município de Santo Antônio de Jardim, aproximadamente 32\% da região, com intenso processo de exploração, sendo utilizada de forma inadequada, sem a adoção de critérios de capacidade de uso.

\section{LITERATURA CITADA}

Bertolini, D.; Lombardi Neto, F. Embasamento técnico do programa estadual de microbacias hidrográficas. In: Lombardi Neto, F.; Drugowich, M.I. (coords.). Manual técnico de manejo e conservação de solo e água. v. 1, CATI. Campinas: CATI, 1994. 65p. Manual Técnico, 38.

Bertoni, J.; Lombardi Neto, F. Conservação do solo. São Paulo: Ícone, 1990. 355p.

Burrough, P.A. Principles of geographical information systems for land resources assessment. New York: Oxford University Press, 1986. 193p.

EMBRAPA - Empresa Brasileira de Pesquisa Agropecuária. Centro Nacional de Pesquisa de Solo. Sistema brasileiro de classificação de solos. Rio de Janeiro: EMBRAPA Solos, 1999. 412p.

Giarratano, J.C.; Riley, G. Expert Systems: principles and programming. 2.ed. Boston: PWS, 1993. 644p.

Giboshi, M.L. Desenvolvimento de um sistema especialista para determinar a capacidade de uso da terra. Campinas: UNICAMP, 1999, 77p. Dissertação Mestrado

Giboshi, M.L.. Sistema de apoio ao processo de decisão para a gestão do uso agrícola da terra. Campinas: UNICAMP, 2005, 114p. Tese Doutorado

Lagrotti, C.A.A. Planejamento agroambiental do município de Santo Antônio do Jardim - SP: Estudo de caso da microbacia hidrográfica do Córrego do Jardim. Campinas: UNICAMP, 2000, 124p. Tese Doutorado

Lepsch, I.F.; Bellinazi Jr., R.; Bertolini, D.; Espíndola, C.R. Manual para levantamento utilitário do meio físico e classificação de terras no sistema de capacidade de uso. 4a Aproximação. 2.ed. Campinas: Sociedade Brasileira de Ciência do Solo, 1991.175p.

Lombardi Neto, F.; Bellinazzi Jr., R. (coords.). Simpósio sobre terraceamento agrícola, 1, 1989, Campinas. Anais... Campinas: Fundação Cargil, 1989. 266p.
Lombardi Neto, F.; Drugowich, M.I. (coords.). Manual técnico de manejo e conservação de solo e água. V. III CATI. Campinas: CATI,1994. P.121-156. Manual Técnico, 40.

Oliveira, J.B. Levantamento pedológico semidetalhado do Estado de São Paulo: quadrícula de Mogi Mirim. São Paulo: IGC, 1992. esc. 1:100.000.

Pereira, L.C. Aptidão agrícola das terras e sensibilidade ambiental: proposta metodológica. Campinas: UNICAMP, 2002. 122p.Tese Doutorado

Plant, R.E.; Stone, N.D. Knowledge-Based Systems in Agriculture. San Francisco: McGraw-Hill, 1991. 364p.

Sartori, A. Avaliação da classificação hidrológica do solo para a determinação do excesso de chuva do método do serviço de conservação do solo dos Estados Unidos. Campinas: UNICAMP, 2004. 161p. Dissertação Mestrado

Sprague, R.H.; Watson, H.J. Decision support systems: putting theory into practice. Englewood Cliffs: Prentice Hall, 2 ed., 1989. 419p.

Sawyer, B.; Foster, D.L. Programming expert systems in Pascal. New York: John Wiley \& Sons, 1986. 186p.

APENDICE: Relatório com recomendação do manejo feitas pelo sistema

Santo Antônio do Jardim

Solo-1: Latossolo Vermelho (LEd3/so - Limeira)

\section{\begin{tabular}{|ll|l|}
\hline $\mathrm{CD}$ & $\mathrm{CCU}$ & LIMITAÇÕES \\
\hline $\mathrm{B}$ & $\mathrm{III}$ & $\mathrm{re} 1 \mathrm{~m} 1 \mathrm{v} 3 \mathrm{f} 2 \mathrm{a} 3$ \\
\hline
\end{tabular}}

Cultura anual - Uso adequado

Recomendações

Rompimento ou desagregação da camada compactada quando esta existir.

Nivelamento do terreno para uma boa performance das máquinas e equipamentos, desde o plantio até a colheit

Plantio em nivel ou em contorno

Tratos culturais normais: controle de ervas daninhas, pragas e doenças.

As operaçőes de preparo do solo, plantio, cultivo e colheita deverăo ser realizadas de maneira apropriada a fim de deixar material residual na superfície ou parcialmente incorporado ao solo. Năo queimar restos de culturas.

o preparo do solo deve ser feito com o menor número possivel de operaçőes, reduzindo a mobilização dos solos, o tempo e o consumo de combustivel necessários.

restos de cultura.

Sucessão de culturas: incluir cultura de inverno e culturas que produzam alta quantidade de material residual.

Culturas em faixas (rotação, retençăo ou conjugadas).

Adubaçăo verde elou orgânica: manter ou aumentar o teor de matéria orgânica do solo.

Recomendaçōes para problemas relacionados à fertilidade

Utizar corretivos e fertilizantes em quantidades adequadas após análise do solo que deve ser repetida com intervalos de no máximo três anos. Manter em arquivo os resultados das análises para acompanhamento da evoluçăo da fertilidade.

Risco de erosăo: ligeiro

solo reduzido.

Adotar o sistema de plantio direto.

\section{Sistema de terraceamento}

O terraceamento não deve ser utilizado como prática isolada no controle da erosão, mas associado a outras práticas conservacionistas, como preparo do solo, plantio e cultivo em nivel, manejo de restos culturais e outras, protegendo as faixas de terra entreterraços.

Terraços em nivel. Base larga.

Construir canais escoadouros como complementos de terraços em nivel com as pontas abertas

Cuidados que devem ser tomados ao se construírem os canais escoadouros

Sua construçăo deve ser anterior a qualquer sistema de captaçăo e conduçăo de excesso de sua cor

Evitar o escoamento contínuo e prolongado de excesso de água no canal (ex.: mina de água), drenando-o por subsuperfície, ou, entấo, protegendo a parte central do canal com

pedra ou concreto.
Reduzir o volume de enxurrada a ser conduzido, dividindo-o para dois ou mais canais.

Manter a cobertura vegetal cortada e adubada, quando necessário

Năo usar voçorocas, areas de propriedades vizinhas, estradas, matas ou pastos como locais de deságüe do canal escoadouro.

Năo permitir manobras de máquinas agricolas sobre o canal, pastoreio de gado, plantas

invasoras e falhas na vegetaçäo.

Manutenção

Após chuvas intensas, os terraços devem ser inspecionados e restaurados se necessário. Manutençấo anual dos terraços e canais escoadouros:

Subsolagem no canal do terraço a cada dois ou três anos para melhorar a infiltraçăo. 